\title{
Capacitive resistivity imaging with towed arrays
}

\author{
Oliver Kuras ${ }^{1}$, Philip I. Meldrum, David Beamish, \\ Richard D. Ogilvy and Deepak Lala
}

British Geological Survey, Kingsley Dunham Centre, Keyworth, Nottingham, NG12 5GG, United Kingdom

${ }^{1}$ Corresponding author, e-mail: O.Kuras@bgs.ac.uk 


\begin{abstract}
The capacitive resistivity (CR) technique is a generalization of the conventional DC resistivity method that facilitates measurements of electrical resistivity on engineered surfaces and highly resistive ground. The CR methodology allows the use of towed sensor arrays, thus enabling the rapid collection of high-resolution resistivity data. Under quasi-static conditions $\mathrm{CR}$ data are equivalent to galvanic DC measurements so that CR datasets can be interpreted with conventional DC inversion algorithms. In this study, we demonstrate that the methodology and fundamental parameters of the CR technique facilitate spatial sampling at the centimeter scale for towing speeds of the order of $2.5 \mathrm{~km} / \mathrm{h}$. We argue that the dipole-dipole array is the most suitable geometry for dynamic CR measurements and present example data acquired with a prototype instrument using plate-wire combinations arranged in an equatorial geometry. The information content of raw CR data is found to be dominant over towing-induced noise and a direct comparison with DC profile data shows good agreement between both techniques. Based on these findings, we show that tomographic imaging is possible using datasets acquired with moving arrays. Closer investigation of the practical aspects of towed-array capacitive resistivity imaging (CRI) highlights the similarities with galvanic multi-electrode surveys, but the different geometric constraints and sampling regime of CRI give rise to advantages (high lateral resolution) as well as disadvantages (limitations in vertical resolution). We conclude our study with recommendations for practical CRI survey procedures and present a field example where we have successfully imaged a subsurface target in 3D. Towed-array CRI is found to provide equivalent (and in some ways superior) information about the shallow subsurface when compared to DC ERT; it therefore widens the scope of electrical
\end{abstract}


imaging surveys to environments where the conventional methodology would be impractical.

\section{Introduction}

Many environmental and engineering site investigations are carried out in the urban built environment, for example on industrial and brownfield sites, derelict and contaminated land, or paved roads and runways. Electrical imaging techniques such as electrical resistivity tomography (ERT) can contribute valuable information to such investigations, as the diagnostic properties of resistivity apply to a wide range of problems. However, typical ground conditions in the built environment often make galvanic measurements impractical and a need has arisen for electrical imaging methodologies better adapted to such conditions. The problems encountered are twofold. Firstly, many sites contain sealed areas with paved surfaces, making the mechanical installation of steel electrodes difficult if not impossible. Even where probes can be deployed, resistivity measurements are at risk of being grossly inaccurate due to the high contact impedances associated with resistive surface materials (Zonge and Hughes, 1986). Secondly, adequate characterization of a complex subsurface requires dense spatial sampling (often at the centimeter scale) and efficient data acquisition procedures. However, high-volume acquisition of resistivity data remains cumbersome despite the increased popularity of multi-electrode/multi-channel instrumentation, as galvanic electrodes must still be manually implanted in the ground.

Capacitive resistivity (CR) is an emerging geophysical technique (Kuras et al., 2006) with the potential to overcome both problems. Suitable instrumentation has been available both at a research level and commercially for some time; however it appears that many basic issues surrounding mobile capacitive measurements have not yet been 
addressed in the published literature. The purpose of the present study is (1) to investigate the use of towed-array CR measurements as an alternative to conventional DC resistivity with regard to practical aspects and the efficiency of data acquisition, (2) to describe the properties of dynamically acquired CR data, and (3) to demonstrate the feasibility of tomographic imaging using datasets acquired with towed arrays.

We begin with a brief discussion of the CR technique itself, after which we investigate the practical aspects of dynamic CR measurements with towed arrays. We review dynamic DC measurement techniques and discuss differences of the capacitive approach, including the influence of towing speed and choice of array geometry. We then describe a prototype instrument with towed sensor arrays and discuss examples of dynamically acquired CR data and their particular characteristics. A direct comparison between static DC and towed-array CR data is presented. Based on our findings, we examine the practical survey procedures and data processing techniques required for capacitive resistivity imaging (CRI), i.e. electrical tomography performed with towed CR arrays. We conclude by presenting a practical field example in which a towed-array CRI survey was undertaken to image a simple subsurface target in 3D.

\section{The capacitive resistivity technique}

Methodology, basic physics and the advantages and limitations of capacitive resistivity were the subject of a recent paper by the same authors (Kuras et al., 2006). The technique is based on a four-point sensor array that is capacitively coupled to the ground (Figure 1) and acts as an oscillating non-grounded electric dipole. This dipole is used as a controlled source to create electric current flow in the ground. Moderate frequencies in the audio range are employed and the CR measurement operates in a quasi-static mode under the condition of low induction numbers as defined by McNeill 
(1980). The coupling mechanism between sensors and the ground is then predominantly capacitive, and inductive effects are negligible. Under quasi-static conditions the capacitive measurement of resistivity is essentially equivalent to the DC method. The objectives are (1) to emulate the DC measurement in such a way that conventional DC survey techniques (resistivity sounding, profiling, tomographic imaging) can be employed with CR, and (2) to ensure that apparent resistivities determined in this fashion comply with traditional DC interpretation schemes such as forward and inverse resistivity modeling.

Capacitive sensors. DC resistivity theory demands that electrical contact between the measuring device and the ground surface is restricted to point poles. A practical realization is the four-point array of metallic stakes typically used in conventional DC surveys. The properties of an idealized, capacitively coupled array can equally be formulated on the basis of point poles, leading to the concept of an “electrostatic quadrupole” (Grard and Tabbagh, 1991). Kuras (2002) has shown that practical realizations in the form of plate-wire combinations (Figure 1), i.e. a flat metallic conductor whose dimensions are small compared to the size of the fourelectrode array and the length of connecting wires, can be designed in such a way that they are compatible with this theory. Coupling is then largely confined to the surface of the plate and, on the scale of the four-point array, the point-pole condition is approximately fulfilled so that a DC geometric factor can be calculated. Another conceivable type of capacitive sensor is the capacitive line antenna (Timofeev et al., 1994), but interpretation of measurements is not trivial as the capacitance of such antennas is evenly distributed across their entire length (Kuras et al., 2006). In this paper, we will therefore focus on the use of plate-wire sensors. 
Quadrupole in quasi-static approximation. We consider an electric quadrupole in the vicinity of the air-earth interface, consisting of two poles carrying slowly varying opposite charges, and two further poles between which a potential difference is being measured. If a current is passed between the former two poles, the arrangement can be regarded as a capacitive four-point array. Following Grard (1990), the transfer impedance of such an arrangement in quasi-static approximation ("electrostatic quadrupole”) can be described as:

$$
Z=\frac{U}{I}=\frac{\hat{U}}{\hat{I}} e^{i \varphi}=Z_{0}\left(1-K^{E S} \alpha\right)
$$

where $U$ is the observed potential, $I$ is the injected current, $Z_{0}=\left(i \omega C_{0}\right)^{-1}, C_{0}$ is a constant, $K^{E S}$ is the electrostatic geometric factor containing sensor elevation (Kuras et al., 2006) and

$$
\alpha=\frac{\rho \omega \varepsilon_{0}\left(\varepsilon_{r}-1\right)-i}{\rho \omega \varepsilon_{0}\left(\varepsilon_{r}+1\right)-i} .
$$

This formalism applies to low induction numbers only and describes the quadrupole response as a function of electrical properties of the subsurface, geometric parameters and the frequency of operation. A practical field technique must determine resistivity from a measurement of the complex impedance. The apparent resistivity measured by a capacitive system is generally a function of both magnitude and phase of Z. However, under quasi-static conditions and if the poles are close to the surface, the quadrature component of $Z$ vanishes and the response can be expected to be in-phase. As a consequence:

$$
\rho_{a} \approx-\frac{\operatorname{Im} \alpha}{2 \omega \varepsilon_{0}} \approx \frac{C_{0}}{2 \varepsilon_{0}} \operatorname{Re} Z=\frac{\operatorname{Re} U}{\hat{I}} K^{D C},
$$


where $K^{D C}$ is the DC geometric factor, i.e. the phase-sensitive apparent resistivity reduces to the classical DC expression for the in-phase component of the transfer impedance (Tabbagh et al., 1993; Kuras et al., 2006).

Significance of the complex measurement. A small number of commercial instruments (e.g., Møller, 2001; Pellerin et al., 2003; Chouteau et al., 2004) and research prototypes (e.g., Shima et al., 1996; Panissod et al., 1998) are currently available for making capacitively coupled resistivity measurements. A list is provided in Kuras (2002). Many of the existing practical designs operate on the basis of an assumed inphase response; hence measurement of the magnitude of the transfer impedance is accepted as sufficient and apparent resistivity is determined by means of the DC formula. However, although such practice may often be justified, quasi-static conditions are generally not sufficient for the CR response to be entirely in-phase (Kuras et al., 2006). Even under quasi-static conditions, the electrostatic formalism provides for circumstances in which the quadrature component of $\mathrm{Z}$ is non-negligible. In such cases, only the in-phase component of the fully complex response must be considered in order to derive an accurate resistivity estimate from a CR measurement.

\section{Dynamic measurements with towed arrays}

Dynamic measurements have been standard practice in electrical and EM methods for many years (e.g. airborne EM, ground penetrating radar). A truly dynamic, continuous acquisition process is the most efficient in terms of ground coverage and can provide densely sampled datasets. However, the use of dynamic acquisition for DC resistivity is limited due to the requirement of maintaining galvanic contact at all times. 
Towed DC arrays. Two classes of systems for making dynamic DC measurements have been described in the literature. One approach is simply to mechanize the procedure of implanting electrodes, for example with spiked metallic wheels (Hesse, 1966) or pneumatically controlled electrodes (Südekum, 2000) that can temporarily establish galvanic contact. The drawbacks of this procedure are that the sampling interval remains fixed and soft ground is required for reliable operation. Another approach, commonly referred to as pulled-array continuous electrical profiling or PA-CEP (Sørensen, 1996), involves dragging an array of heavy metallic electrodes across the ground surface in an attempt to permanently maintain galvanic contact. Current injection and potential measurement can then be carried out simultaneously and continuously. A hybrid technique involves the use of high-pressure jets of conductive liquid injected continuously into the ground to provide galvanic coupling (Hesse et al., 1986). The major disadvantage of towed-array DC resistivity measurements is that data tend to be affected by high levels of motion-induced noise due to temporary variations in galvanic contact (Munkholm, 1996; Sørensen, 1996). On artificial ground, conditions are usually worse due to high contact impedances, making dynamic DC techniques less suitable for surveys in the built environment.

Towed capacitive arrays. Key advantages of CR are that (1) the measurement is unaffected by the nature of the surface material, (2) high signal-to-noise ratios are achievable in resistive environments and (3) the technique facilitates a dynamic mode of operation, in which data are acquired continuously from a towed sensor array. Such arrays consist of multiple capacitive sensors (current and potential dipoles) in a fixed geometrical arrangement, mounted on some kind of mobile mechanical platform, for example a trailer, frame, mat or carpet (Panissod et al., 1998). The entire unit is then 
pulled along manually by an operator or towed by a vehicle, so that sensors are moving at constant separation. Multi-dipole arrays with increasing offsets enable multi-depth resistivity mapping and the efficient collection of tomographic datasets in a single pass of the instrument. The potential increase in productivity over single-dipole acquisition is obvious. As towed-array surveys are necessarily conducted along linear profiles, progress is always successive, i.e., different parts of the survey area are covered consecutively. Reciprocity allows towed-array CRI surveys to be bi-directional, so that data may be acquired in different orientations on neighboring profiles.

Practical aspects of continuous sampling. Practical data acquisition techniques imply that sampling intervals along the survey profile tend to be regular either in time or space. Constant-frequency sampling produces data at regular intervals in time. The usual survey procedure is then to record marker points (fiducials) at regular intervals over the desired distance, between which sampled data are then distributed equally through linear interpolation. The disadvantage of this approach is that spatial sampling along the survey profile may become irregular if the towing speed varies.

In most near-surface applications it is desirable to sample dynamically acquired data at constant spacing $\Delta s$ throughout a survey area, if no prior information about the subsurface is available. Moreover, many data processing and interpretation schemes call for, or benefit from, equally spaced data (e.g. filtering, spatial Fourier analysis, resistivity modeling and inversion algorithms). In our implementation of the towedarray CR technique we therefore employ regular sampling in space in order to avoid potential loss of information through re-sampling at a later stage. 
Time scale of the measurement and implications for towing speed. Dynamic spatial sampling at the centimeter scale requires the ability to make very rapid measurements. For example, at typical towing speeds for mobile arrays of $2.5 \mathrm{~km} / \mathrm{h}$, a distance of the order of $1 \mathrm{~cm}$ on the ground corresponds to a time of $\tau \approx 14 \mathrm{~ms}$ during which a single measurement must be performed while the array is moving at constant speed. The horizontal sampling interval cannot be arbitrarily small as it is limited by the time scale $\tau$ of the measurement process itself. This time constant is linked to the frequency of operation and depends on the measurement principle.

In the case of DC resistivity, low-frequency AC or switched DC signals are commonly employed to avoid compensation for electrode self-potential (Hesse et al., 1986). For switched DC, measured voltage waveforms are then evaluated after a delay time following each flank when the potential is assumed to have reached a plateau. Typical cycle times are of the order of seconds. For dynamic DC measurements, spatial sampling at the centimeter scale therefore requires cycle times as short as $8 \mathrm{~ms}$ (corresponding to a frequency of $125 \mathrm{~Hz}$ ) and the construction of resistivity meters that can make a single measurement per cycle (Hesse et al., 1986).

In the case of CR, cycle times are significantly smaller due to the use of audio frequencies (e.g. $66.7 \mu \mathrm{s}$ at $15 \mathrm{kHz}$ ). The same time constant of $\tau \approx 14 \mathrm{~ms}$ corresponds to hundreds of current cycles (e.g. 210 cycles at $15 \mathrm{kHz}$ ). In our implementation of the CR measurement, we employ phase-sensitive signal detection to extract magnitude and phase of the complex transfer impedance. This concept requires the availability of signal waveforms containing many current cycles for each individual measurement. Based on the above parameters, spatial sampling at the centimeter scale is therefore possible with our methodology. 
As a consequence of the finite time required for individual measurements during towed-array CR data acquisition, readings are not actually obtained at a fixed position in space but over a small distance. This fundamental averaging effect is inherent to dynamic measurements. However, even greater spatial averaging is associated with the finite size of the capacitive sensors. The electric potential is determined for an area of ground corresponding to the size of the individual sensor. In the case of our capacitive plates, this means that individual readings represent an average over the plate width.

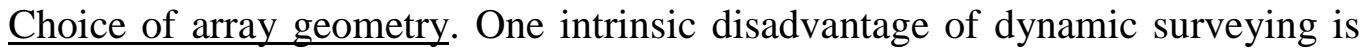
that the number of potential dipoles that can be installed on a towed array is limited and the geometric configuration must remain constant. It is therefore important to choose a geometry that is consistent with the character of the investigation. Some electrode configurations are generally unsuitable for towed capacitive arrays. The use of remote electrodes (pole-pole and pole-dipole arrays) is common practice in DC resistivity and such arrays have been used for mobile DC surveying (Panissod et al., 1998). However, the length of wire connections to the remote poles prohibits their use on towed capacitive arrays due to the large capacitances associated with long wire segments. Wenner and Schlumberger array configurations are also problematic due to the location of the potential dipole between the current electrodes. In our experience, such an arrangement tends to be susceptible to cross-talk between transmitter and receiver wires as the wiring from all dipoles would have to run along the axis of the array.

Our work has shown that the dipole-dipole array is the most suitable geometry for dynamic CR measurements. Mutual coupling between transmitter and receiver wires is avoided entirely, particularly if the transmitter is placed at the rear end of the array. The necessary phase reference from the current source can be obtained by fiber optic 
transmission. The two elementary dipole-dipole configurations are the inline and equatorial varieties (Parasnis, 1996). DC sensitivity analysis (Spitzer, 1998) reveals differences in their spatial properties. 3D sensitivity distributions calculated for a homogeneous halfspace are shown in Figure 2 for both configurations. The near-vertical contour pattern in the vertical cross-section along the towing direction (Figure 2b) indicates that the inline configuration is sensitive to lateral changes in resistivity along the survey profile, but less sensitive to vertical changes (Loke, 2004). The equivalent contour pattern for the equatorial array suggests that this configuration is likely to be more sensitive to lateral changes on either side of the survey profile (Figure 2c).

A clear disadvantage of the dipole-dipole array is the fact that observed potentials are very small at large separations (i.e. large $n$-factors). The inline geometry represents the configuration of maximum coupling between current and potential dipoles. The coupling in the equatorial configuration is weaker; hence a weaker signal is observed at comparable dipole separations. A remedy for this problem is to increase the transmitter dipole moment by using larger dipoles or stronger currents or to use larger potential dipoles in order to obtain a stronger signal. The latter approach was followed by Panissod et al. (1997), who have employed an equatorial array in which the length of the potential dipoles increases with distance from the current dipole.

From experience with near-surface investigations using towed CR arrays, we have developed a preference for the equatorial configuration for the following reasons:

- Relative to its overall array size, the equatorial array has by far the largest median depth of investigation compared to other standard array configurations (Edwards, 1977).

- Towed multi-channel arrays must be easy to handle and maneuver, particularly when deployed on sites in the built environment where space is often limited. The 
nature of the equatorial geometry allows the construction of compact multi-offset arrays.

\section{Prototype instrumentation and comparison with DC data}

In the following we present towed-array datasets acquired with a newly developed instrument. This so-called CRI (Capacitive Resistivity Imaging) system has been designed and built by the British Geological Survey (BGS) and is a straightforward implementation of the theoretical concept of an electrostatic quadrupole. Its major improvement over previous designs is that it is capable of measuring the full complex transfer impedance across pairs of capacitive sensors without the need for a fixed reference potential. This concept significantly reduces towing noise observed with existing CR instruments. The electronic design of the CRI system is based upon modern signal detection technology. It is designed for synchronous acquisition from multiple receiver channels and can perform both static and dynamic CR measurements (Beamish et al., 2001; Kuras, 2002).

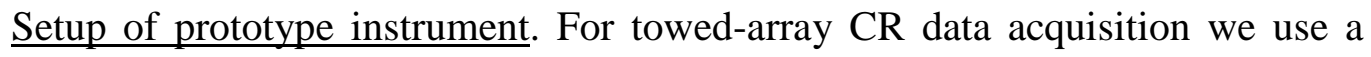
configuration consisting of one current dipole and up to six potential dipoles in an equatorial dipole-dipole configuration with a typical dipole length of $1.5 \mathrm{~m}$ and separations ranging from $1.6 \mathrm{~m}$ to $6 \mathrm{~m}$. The capacitive plates are encapsulated in plastic sheeting for mechanical protection and electrical insulation. The system is pulled by an off-road vehicle (Figure 3). Equally spaced data are obtained by triggering the acquisition at predefined spatial intervals. The distance measurement is realized by an odometer attached to the sensor array or towing vehicle. Alternatively, we have successfully tested data acquisition controlled by Real-Time Kinematic GPS (Strange, 
2003). For practical reasons, we choose the sampling interval to be identical for all receiver channels, i.e. short-offset data are obtained at the same $\Delta s$ as long-offset data.

Properties of dynamically acquired CR data. We have undertaken a range of test surveys making dynamic measurements in a variety of environments to examine the dynamic properties of the CR transfer impedance and to assess the data quality obtained under different survey conditions. A typical dataset recorded on grass is shown as an example in Figure 4. The site is underlain by solid limestone covered by a thin layer of topsoil. The data are unfiltered and were acquired using a dipole separation of $r=1.6 \mathrm{~m}$ and a sampling interval of $\Delta s \approx 6 \mathrm{~cm}$. Although the recorded transfer impedance profile is very dynamic, it appears to contain low proportions of towing noise. Typical indications for noisy data such as single outliers (spikes) are very rare (Figure 4a-c). The injected current (Figure 4d) varies between approximately 7 and $8 \mathrm{~mA}$; the actual values are recorded and used in the impedance calculation. Apparent resistivity values (Figure 4e) range from approximately $70 \Omega \mathrm{m}$ to $175 \Omega \mathrm{m}$. The limestone on the site is known to be fractured and contains a number of manmade cavities, thought to be associated with a number of well-defined resistivity anomalies ( $\mathrm{x}=19 \mathrm{~m}, 28 \mathrm{~m}$ and $34 \mathrm{~m}$ ). The median depth of investigation of the array (Edwards, 1977) is approximately $0.71 \mathrm{~m}$. Although the available ground truth information is insufficient to make this a controlled experiment, we consider this dataset to be a good indication for the capabilities of towed-array CR under field conditions.

Comparison with DC data. An important aim of the present study was to directly compare dynamically acquired CR data with conventional static DC resistivity measurements. To achieve this, we have acquired CR and DC resistivity data with 
identical array geometries on the same traverse over a shallow buried target on a grasscovered field at the BGS site in Keyworth. Our prototype CRI system and a conventional multi-electrode DC resistivity instrument were used for this purpose. In order to simulate the equatorial geometry of the CRI array, two rows of stainless steel electrodes with a separation of $1.5 \mathrm{~m}$ were installed parallel to the survey line. A unit spacing of $0.4 \mathrm{~m}$ was used for the DC electrodes, which allowed us to emulate a capacitive equatorial array with $1.6 \mathrm{~m}$ separation. The DC instrument was then programmed to measure apparent resistivities consecutively across subsequent pairs of dipoles with constant separation along the survey line. A resistivity profile with a sampling distance of $0.4 \mathrm{~m}$ was obtained. Such coverage is relatively dense for typical site investigation surveys with DC resistivity, yet the resolution achievable with CR data is higher still. In our case, the sampling interval of $\Delta s \approx 5 \mathrm{~cm}$ corresponds to eight CRI samples per DC electrode spacing. Figure 5 shows the results of this experiment. The buried target is a large concrete cube (side length $1.5 \mathrm{~m}$ ) cast in situ at a depth of $0.5 \mathrm{~m}$ below the ground surface (distance to the top of the concrete). The center of the cube is located at a profile distance of approximately $15 \mathrm{~m}$. The overall shape of the central $\rho_{a}$ anomaly is very similar for both techniques, yet the additional detail contained in the CRI data is obvious. Small offsets in magnitude between both curves exist, but do not appear to be consistent along the profile. Both datasets are compatible and relative amplitudes of the $\rho_{a}$ maximum over the target are consistent for both techniques. Small deviations may be associated with geometric differences due to the finite size of the capacitive sensors. 


\section{Towed-array capacitive resistivity imaging}

The above results suggest that multi-offset towed-array CR data can indeed be used for tomographic imaging. The equivalence between apparent resistivities determined by the DC and CR methods facilitates the application of conventional DC resistivity forward modeling and inversion algorithms to CRI data. This is straightforward if some basic rules are observed with regard to array geometries and survey design (Kuras, 2002). At the simplest level, continuous vertical electrical soundings (CVES) may be extracted from such data, which may form the basis of a quasi-2D interpretation (Kuras, 2003b). Full 2D or 3D resistivity inversion of towedarray CRI data presents a number of challenges to survey design and data processing. In the following, we discuss the differences compared to DC resistivity imaging and provide an example for the 3D interpretation of a CRI survey over a simple subsurface target.

Fixed-electrode acquisition. It is standard practice to acquire multi-offset DC resistivity data with automated multi-channel systems using lines or grids of fixed electrodes spaced at regular intervals (Griffiths and Turnbull, 1985; Griffiths et al., 1990; Dahlin, 2001). Current and potential electrodes are moved along the profile by activating consecutive electrode positions along a multi-core cable. The consequences of this methodology are:

- The lateral sampling distance for a given array geometry is constant, i.e. the ground is probed at regular intervals along the profile. Consequently, apparent resistivity datum points follow a regular pattern in the distance-pseudodepth plane, typically with triangular or trapezoidal shape (Figure 6a). 
- Dipole size and array separation are always integer multiples of the fundamental electrode spacing. Hence, vertical sampling of the subsurface achieved by extending the electrode spread is also discrete and limited to well-defined median depths of investigation.

- Lateral and vertical sampling of the subsurface are interdependent and a function of the fundamental electrode spacing $d$.

These properties of fixed-electrode data are often used in interpretation schemes. The use of regular electrode spacings entails a laterally regular discretisation of model grids (Barker, 1992) for forward and inverse modeling, although vertical discretisation can be non-uniform. Some filtering techniques also make use of equally spaced data (Acworth and Griffiths, 1985).

Towed-array acquisition. In contrast, towed-array data (DC as well as CR) may lack such convenient spatial reference. Whilst the geometry of the towed electrode array remains constant, its position on the ground is variable. A key step in the processing of multi-offset towed-array CR data is therefore to establish the locations of individual measurements and allocate apparent resistivities acquired from the moving array, so that readings can be accurately positioned in the distance-pseudodepth plane. Characteristics of the towed-array approach are:

- Compared to DC resistivity, the lateral sampling distance can be far smaller than the overall dimensions of the electrode array and may vary with the towing speed along the profile if constant-frequency sampling is employed (Figure 6b).

- The relation between the sampling interval and the dipole offsets determines the distribution of datum points in the pseudosection. The geometry can be chosen such 
that this distribution becomes regular, provided that constant-distance sampling is employed (Figure 6c).

- The distribution has the shape of a parallelogram, assuming that the current dipole is located at one end of the array (Figure 6c).

- Towed array data may be less interdependent than fixed-electrode data since array size and separations do not have to be related to a fundamental spacing.

Despite this greater flexibility, it is still possible to employ conventional DC interpretation schemes to handle multi-offset towed-array CR data. If towed-array surveys are planned with care, data processing can be kept to a minimum and unnecessary re-sampling can be avoided. In this paper we assume that all dipole offsets are integer multiples of the sampling interval. For all other cases, the alternative is to resample individual apparent resistivity channels so that lateral coincidence is achieved.

3D towed-array survey design and techniques. We perform 3D data acquisition with towed CR arrays along a sequence of linear profiles (Figure 7) and obtain volumetric coverage by conducting multi-offset measurements on parallel survey lines. Due to reciprocity (Parasnis, 1988), the towed array may be orientated in either direction along the profiles. For towed-array CRI, sampling intervals can be significantly different in the in-line and cross-line directions (Figure 7c). Acquiring data in this fashion on survey lines in orthogonal direction may result in "blank" areas of ground that have no datum points associated with them (Figure 7d). Although collecting data with independent array orientations may contribute to improved definition of complex targets, we have found 3D interpretation of datasets with orthogonal profiles and a regular mesh of model cells to be unrealistic due to the potentially large number of model parameters required in relation to the number of datum points obtained by 
irregular coverage of the ground. Such datasets would most likely still have to be degraded by downsampling onto a coarser grid.

We have used the equatorial array for 3D data acquisition, which has further implications for 3D interpretation. Application of conventional DC inversion algorithms based on a regular 3D mesh implies that the towed-array data have to be sampled in a manner that corresponds to data collected with a regular grid of conventional DC electrodes. Line spacings must be chosen such that electrode positions on adjacent survey lines are compatible with electrode positions in the 3D mesh. In order to avoid resampling, the simplest approach is to design the towed-array survey accordingly. Starting the survey at a common baseline ensures lateral coincidence of the sampling locations in profile direction. For an equatorial array, coincidence of electrode positions in the cross-profile direction can be achieved by choosing a line separation that has a common denominator with the dipole length of the equatorial array. An example is shown in Figure 8, where the line spacing is $1 / 3$ of the dipole length of a towed array with equatorial geometry.

The practical realization of 3D tomographic imaging surveys with towed CR arrays involves navigating the combination of towing vehicle and sensor array over a sequence of survey lines with a positional accuracy of the order of centimeters. The higher efficiency of the dynamic measurement is a key advantage of CRI and must not be compromised by delays due to positioning and navigation. Our experience has shown that the most efficient method of acquiring data on parallel survey lines is by bidirectional surveying, where adjacent lines are surveyed in opposite directions and the vehicle u-turned at the end of each line, thus following a continuous "snake path". Unidirectional surveying requires long periods of downtime due to lengthy repositioning. In an effort to increase spatial accuracy as well as survey speed, GPS systems (Strange, 
2003) or automated surveying equipment such as self-tracking laser theodolites (Lehmann and Green, 1999) can be employed for real-time navigation on a survey grid. We have successfully tested Real-Time Kinematic GPS navigation in conjunction with towed-array CRI (Kuras, 2003a).

\section{D tomographic imaging: a field example}

In order to demonstrate the practicality of tomographic imaging under field conditions, we have undertaken a towed-array CRI survey at the Southmeads Road test site at the University of Leicester, UK. The site is operated by the Environmental and Industrial Geophysics Group (EIGG) of the Geological Society of London and is described in detail by Hill (2000). It comprises a variety of buried environmental and engineering targets made of a range of different materials and installed under controlled conditions. The site lies on the summit of a gentle hilltop and is characterized by a relatively uniform surface layer of clayey topsoil, which is approximately $0.3 \mathrm{~m}$ thick, grading down into Boulder Clay. The latter unit is uniform throughout the site, 16-18 m thick and underlain by Liassic clays and limestones. Conventional DC resistivity soundings suggest a bulk resistivity of the Boulder Clay of approximately $23 \Omega \mathrm{m}$. The water table is shallow, but the clay is of low permeability. The target selected for the survey is known as Area 8 and consists of a sand pit containing small metallic objects. The pit is buried under a layer of topsoil; its layout and constructional details are shown in Figure 9. While the metallic objects are too small to have a significant effect on resistivity measurements at the surface, the resistive sand provides good electrical contrast with the surrounding Boulder Clay and was therefore selected as the main target. The pit is $6 \mathrm{~m}$ long, $2 \mathrm{~m}$ wide and $1.7 \mathrm{~m}$ deep and is covered by $0.3 \mathrm{~m}$ of topsoil. Its long axis is aligned with the $y$-direction of the local coordinate system, which is 
approximately orientated south-north. The pit extends between $57 \mathrm{~m}$ and $59 \mathrm{~m}$ in $x$ direction and $17 \mathrm{~m}$ and $23 \mathrm{~m}$ in y-direction in local coordinates (Figure 9).

Using the CRI system towed by a Land Rover, eleven parallel profiles at a separation of $0.5 \mathrm{~m}$ were acquired over Area 8 . As the site conditions did not allow for the survey to be conducted in a bi-directional fashion, all profiles were acquired in positive $y$-direction (south-north). A sampling interval of $0.25 \mathrm{~m}$ along the survey line was chosen, resulting in a regular virtual grid of electrode positions with $31 \times 105$ electrodes. Recorded apparent resistivities were of high overall quality, so that data preprocessing could be kept to a minimum. Individual profiles were filtered with a predictor filter to remove sporadic outliers and high spatial wavenumber content of the data. All profiles were then collated into one single dataset and inverted. We have employed smoothness-constrained least-squares inversion due to the popularity of this type of algorithm in conventional DC interpretation (Loke and Barker, 1996). The dataset comprised a total number of 5,346 datum points and a 7-layer 3D model with a $0.25 \mathrm{~m}$ discretisation was generated. The inversion process was stopped after six iterations, by which time the RMS error had been reduced to $2.77 \%$.

The resulting 3D resistivity model of Area 8 is shown in the form of stacked horizontal sections in Figure 10. White lines indicate the outline geometry of the sand pit. The surface layer is found to be predominantly resistive (>100 $\Omega \mathrm{m})$, but generally of high variability. Coherent structures are difficult to observe in this layer. Layer 2 is still largely resistive (>50 $\Omega \mathrm{m})$ and patchy, yet a coherent resistive feature associated with the top of the sand pit begins to appear in the centre of the section. From layer 3 onwards, the structure is fully resolved and sharply delimited against the conductive background. The Boulder Clay surrounding the pit is found to be associated with resistivities of the expected order (20-30 $\Omega \mathrm{m})$. Particularly at medium and larger depths, 
the resistive structure is surrounded by a conductive halo (20 $\Omega \mathrm{m}$ and below), possibly as a side effect of the Occam-type inversion method. Despite the smoothness constraint, the geometric definition of the target is precise, due largely to the high spatial resolution of the dataset and model. The only shortcoming of the inverted model is the lack of definition at depth. The bottom of the sand pit $(2.0 \mathrm{~m})$ is clearly not well resolved as the resistive feature is still prominent in the lowest model layer (2.30 m-2.94 m), masking the underlying Boulder Clay. The longest array offset (6 m at Rx 6) corresponds to a maximum effective depth (Edwards, 1977) of only $2.33 \mathrm{~m}$, which is unlikely to be sufficient to accurately resolve the bottom of the pit at $2.0 \mathrm{~m}$.

\section{Conclusions}

Our work has provided evidence that the CR technique is capable of complementing the conventional DC resistivity method. CR facilitates resistivity measurements in the built environment and other settings where galvanic contact cannot be achieved. We have shown both conceptually and experimentally that reliable highdensity dynamic measurements of the fully complex transfer impedance are possible with towed CR arrays based on multiple dipoles of plate-wire combinations. Such arrays were found to provide good quality data at towing speeds up to $2.5 \mathrm{~km} / \mathrm{h}$ with centimeter resolution and high signal-to-noise ratios, even under difficult surface conditions. Our methodology allows for efficient suppression of towing-induced noise and in many circumstances may therefore be more suitable than towed DC arrays. CR generates datasets that appear to contain useful information down to the scale of the sampling interval. Such data may allow the resolution of small-scale variability of the electrical properties in the very near surface. 
We have demonstrated that CR can emulate the DC measurement in such a way that conventional DC survey techniques (resistivity sounding, profiling, tomographic imaging) can be employed with CR, and apparent resistivities determined by capacitive measurement comply with DC interpretation schemes. It was shown that CR has essentially the same tomographic imaging capability as DC resistivity, yet at data densities that are superior to what is normally achievable with DC methods. Such datasets can lead to increased model resolutions and thus help improve target definition. We have shown how CRI can be employed to image subsurface targets in 3D using multi-offset towed arrays.

Our results suggest that the scope for the application of electrical imaging methodologies to environmental and engineering site investigations is widened by CRI. On sites where resistivity tomography would previously have been impractical, and in combination with modern on-site positioning and navigation techniques, towed-array CRI provides a powerful tool for obtaining detailed electrical images of the shallow subsurface. In our opinion, CRI may be best suited for the evaluation of engineered structures such as roads and pavements.

\section{Acknowledgments}

The authors wish to thank Andreas Weller and an anonymous reviewer for their comments, which have helped improve the original manuscript. We gratefully acknowledge the Environmental and Industrial Geophysics Group of the Geological Society of London and Dr Ian Hill of the University of Leicester (UK) for granting access to the Southmeads Road test site. This paper is published with the permission of the Executive Director of the British Geological Survey (NERC). 


\section{References}

Acworth, R.I. and Griffiths, D.H., 1985. Simple data processing of tripotential apparent resistivity measurements as an aid to the interpretation of subsurface structure. Geophys. Prosp., 33: 861-887.

Barker, R.D., 1992. A simple algorithm for electrical imaging of the subsurface. First Break, 10(2): 53-62.

Beamish, D., Meldrum, P.I., Kuras, O. and Ogilvy, R.D., 2001. A new capacitively coupled resistivity system for the electrical impedance imaging of engineered structures, 7th meeting of the Environmental and Engineering Geophysical Society - European Section, Birmingham.

Chouteau, M., Vallieres, S. and Miralles, M., 2004. Assessment of continuous resistivity profiling for the characterisation of paved roads. Eos, Transactions of the American Geophysical Union, Joint Assembly Supplement, 85(17): Abstract NS43A-03.

Dahlin, T., 2001. The development of DC resistivity imaging techniques. Computers and Geosciences, 27(9): 1019-1029.

Edwards, L.S., 1977. A modified pseudosection for resistivity and IP. Geophysics, 42(5): 1020-1036.

Grard, R., 1990. A quadrupolar array for measuring the complex permittivity of the ground: application to Earth prospection and planetary exploration. Meas. Sci. Technol., 1: 295-301.

Grard, R. and Tabbagh, A., 1991. A Mobile Four-Electrode Array and Its Application to the Electrical Survey of Planetary Grounds at Shallow Depths. J. Geophys. Res., 96(B3): 4117-4123. 
Griffiths, D.H. and Turnbull, J., 1985. A multi-electrode array for resistivity surveying. First Break, 3(7): 16-20.

Griffiths, D.H., Turnbull, J. and Olayinka, A.I., 1990. Two-dimensional resistivity mapping with a computer-controlled array. First Break, 8(4): 121-129.

Hesse, A., 1966. Perfectionnement des applications archéologiques de la prospection électrique. Comptes Rendus Mensuels de la Société Préhistorique Française, LXIII: 15-19.

Hesse, A., Jolivet, A. and Tabbagh, A., 1986. New prospects in shallow depth electrical surveying for archaeological and pedological applications. Geophysics, 51(3): $585-594$

Hill, I.A., 2000. Environmental and Industrial Geophysics Group (EIGG) Test Sites, www.le.ac.uk/geology/iah/research/EIGG/eigghp.html.

Kuras, O., 2002. The capacitive resistivity technique for electrical imaging of the shallow subsurface. Ph.D. Thesis, University of Nottingham.

Kuras, O., Beamish, D., Meldrum, P.I. and Ogilvy, R.D., 2006. Fundamentals of the capacitive resistivity technique. Geophysics, 71(3): G135-G152.

Kuras, O., Beamish, D., Meldrum, P.I., Ogilvy, R.D., Strange, K., Waller, M., Roberts, G.W. and Williams, G.M., 2003a. Detection of abandoned mineshafts using towed-array capacitive resistivity and real-time kinematic GPS navigation, 9th Meeting of the Environmental and Engineering Geophysical Society - European Section, Prague.

Kuras, O., Ogilvy, R. D., Beamish, D., Meldrum P. I. and Nathanail, C. P., 2003b. Capacitive Resistivity Imaging with Towed Arrays, Symposium on the Application of Geophysics to Engineering and Environmental Problems (SAGEEP). EEGS, San Antonio, Texas. 
Lehmann, F. and Green, A.G., 1999. Semiautomated georadar data acquisition in three dimensions. Geophysics, 64: 719-731.

Loke, M.H., 2004. Tutorial: 2-D and 3-D electrical imaging surveys. Geotomo Software, www.geoelectrical.com.

Loke, M.H. and Barker, R.D., 1996. Practical techniques for 3D resistivity surveys and data inversion. Geophys. Prosp., 44: 499-523.

McNeill, J.D., 1980. Electromagnetic terrain conductivity measurement at low induction numbers. Technical Note TN-6, Geonics Ltd.

Møller, I., 2001. OhmMapper field tests at sandy and clay till sites in Denmark, 7th Meeting of the Environmental and Engineering Geophysical Society - European Section, Birmingham, pp. 100-101.

Munkholm, M.S., 1996. Robust methods for transient electromagnetic and pulled array geoelectrical data processing. Ph.D. Thesis, University of Aarhus.

Panissod, C., Dabas, M., Hesse, A., Jolivet, A., Tabbagh, J. and Tabbagh, A., 1998. Recent developments in shallow-depth electrical and electrostatic prospecting using mobile arrays. Geophysics, 63(5): 1542-1550.

Panissod, C., Dabas, M., Jolivet, A. and Tabbagh, A., 1997. A novel mobile multipole system (MUCEP) for shallow (0-3 m) geoelectrical investigation: the 'Vol-decanards' array. Geophys. Prosp., 45: 983-1002.

Parasnis, D.S., 1988. Reciprocity Theorems in Geoelectric and Geoelectromagnetic Work. Geoexploration, 25: 177-198.

Parasnis, D.S., 1996. Principles of Applied Geophysics. Chapman \& Hall, London.

Pellerin, L., Groom, D. and Johnston, J., 2003. Multi-receiver OhmMapper survey over a former fuel tank site, 9th Meeting of the Environmental and Engineering Geophysical Society - European Section, Prague. 
Shima, H., Sakashita, S. and Kobayashi, T., 1996. Developments of non-contact data acquisition techniques in electrical and electromagnetic explorations. J. Appl. Geophys., 35: 167-173.

Sørensen, K., 1996. Pulled Array Continuous Electrical Profiling. First Break, 14(3): 85-90.

Spitzer, K., 1998. The three-dimensional DC sensitivity for surface and subsurface sources. Geophys. J. Int., 134: 736-746.

Strange, K.L., 2003. The integration of ground resistivity with GPS. Ph.D. Thesis, University of Nottingham.

Südekum, W., 2000. Mobile Elektrodengruppe zur oberflächennahen geoelektrischen Kartierung. Geologisches Jahrbuch, E52: 35-62.

Tabbagh, A., Hesse, A. and Grard, R., 1993. Determination of electrical properties of the ground at shallow depth with an electrostatic quadrupole: field trials on archaeological sites. Geophys. Prosp., 41: 579-597.

Timofeev, V.M., Rogozinski, A.W., Hunter, J.A. and Douma, M., 1994. A new ground resistivity method for engineering and environmental geophysics, Symposium on the Application of Geophysics to Engineering and Environmental Problems (SAGEEP). EEGS, pp. 701-715.

Zonge, K.L. and Hughes, L.J., 1986. Effect of Electrode Contact Resistance on Electric Field Measurements. Geophysics, 51(2): 463. 


\section{FIGURES}

Figure 1: Basic concept of DC and capacitive resistivity measurements. Left: DC fourpoint array with galvanic electrodes. Right: four-point array with capacitive coupling.

Figure 2: 3D distribution of DC sensitivities for the inline (left) and equatorial (right) configurations of the dipole dipole array. (a) Plan view, (b) cross-section through $\mathrm{y}=0 \mathrm{~m},(\mathrm{c})$ cross-section through $\mathrm{x}=-2.5 \mathrm{~m}$.

Figure 3: The five-channel towed-array CRI system in field operation on a tarmac road.

Figure 4: Typical example of a towed-array CR apparent resistivity profile acquired on grass with dipole separation $r=1.6 \mathrm{~m}$, dipole length $l=1.5 \mathrm{~m}$, sampling interval $\Delta \mathrm{s}=6$ cm; (a) complex potential (in-phase and quadrature), (b) magnitude, (c) phase, (d) current, (e) apparent resistivity.

Figure 5: Direct comparison between apparent resistivity profiles measured with DC resistivity and towed-array CR over a buried concrete cube (side length $1.5 \mathrm{~m}$ ) centered at $x=15 \mathrm{~m}$.

Figure 6: Distribution of datum points in the distance-pseudodepth plane as obtained with fixed-electrode and towed-array data acquisition using a multi-channel array with equatorial dipole-dipole geometry. (a) Fixed-electrode acquisition with constant electrode spacing $d$; (b) towed-array acquisition with constant array spacing $d$, but variable sampling interval $\Delta s$ as obtained by constant-frequency sampling; (c) towedarray acquisition with constant sampling interval $\Delta s$ as obtained by constant-distance sampling. 
Figure 7: Coverage of a regular grid of electrode/sampling positions by parallel survey lines in one single or two perpendicular orientations. (a), (b) Equal or similar sampling intervals in both directions as typical for DC surveys; (c), (d) smaller sampling intervals in in-line direction and larger line spacings as typical for towed-array CR.

Figure 8: 3D towed-array CRI survey on parallel profiles resulting in a regular grid of electrode positions and sampling locations. Black dots represent sensor positions, crosses show datum points. (a) Array position for first line and first sample; (b) second line, first sample; (c) second line, second sample.

Figure 9: Layout of Area 8 of the EIGG test site at the University of Leicester. (a) Plan view; (b) vertical cross-section through the center of the sand pit.

Figure 10: 3D resistivity model (stacked horizontal sections) of Area 8 of the EIGG test site. White lines indicate the outline geometry of the sand pit. 
Figure 1: Basic concept of DC and capacitive resistivity measurements. Left: DC fourpoint array with galvanic electrodes. Right: four-point array with capacitive coupling.

Figure 2: 3D distribution of DC sensitivities for the inline (left) and equatorial (right) configurations of the dipole dipole array. (a) Plan view, (b) cross-section through $\mathrm{y}=0 \mathrm{~m}$, (c) cross-section through $\mathrm{x}=-2.5 \mathrm{~m}$.

Figure 3: The five-channel towed-array CRI system in field operation on a tarmac road.

Figure 4: Typical example of a towed-array CR apparent resistivity profile acquired on grass with dipole separation $r=1.6 \mathrm{~m}$, dipole length $l=1.5 \mathrm{~m}$, sampling interval $\Delta \mathrm{s}=6$ cm; (a) complex potential (in-phase and quadrature), (b) magnitude, (c) phase, (d) current, (e) apparent resistivity.

Figure 5: Direct comparison between apparent resistivity profiles measured with DC resistivity and towed-array CR over a buried concrete cube (side length $1.5 \mathrm{~m}$ ) centered at $x=15 \mathrm{~m}$.

Figure 6: Distribution of datum points in the distance-pseudodepth plane as obtained with fixed-electrode and towed-array data acquisition using a multi-channel array with equatorial dipole-dipole geometry. (a) Fixed-electrode acquisition with constant electrode spacing $d$; (b) towed-array acquisition with constant array spacing $d$, but variable sampling interval $\Delta s$ as obtained by constant-frequency sampling; (c) towedarray acquisition with constant sampling interval $\Delta s$ as obtained by constant-distance sampling.

Figure 7: Coverage of a regular grid of electrode/sampling positions by parallel survey lines in one single or two perpendicular orientations. (a), (b) Equal or similar sampling intervals in both directions as typical for DC surveys; (c), (d) smaller sampling intervals in in-line direction and larger line spacings as typical for towed-array CR. 
Figure 8: 3D towed-array CRI survey on parallel profiles resulting in a regular grid of electrode positions and sampling locations. Black dots represent sensor positions, crosses show datum points. (a) Array position for first line and first sample; (b) second line, first sample; (c) second line, second sample.

Figure 9: Layout of Area 8 of the EIGG test site at the University of Leicester. (a) Plan view; (b) vertical cross-section through the center of the sand pit.

Figure 10: 3D resistivity model (stacked horizontal sections) of Area 8 of the EIGG test site. White lines indicate the outline geometry of the sand pit. 


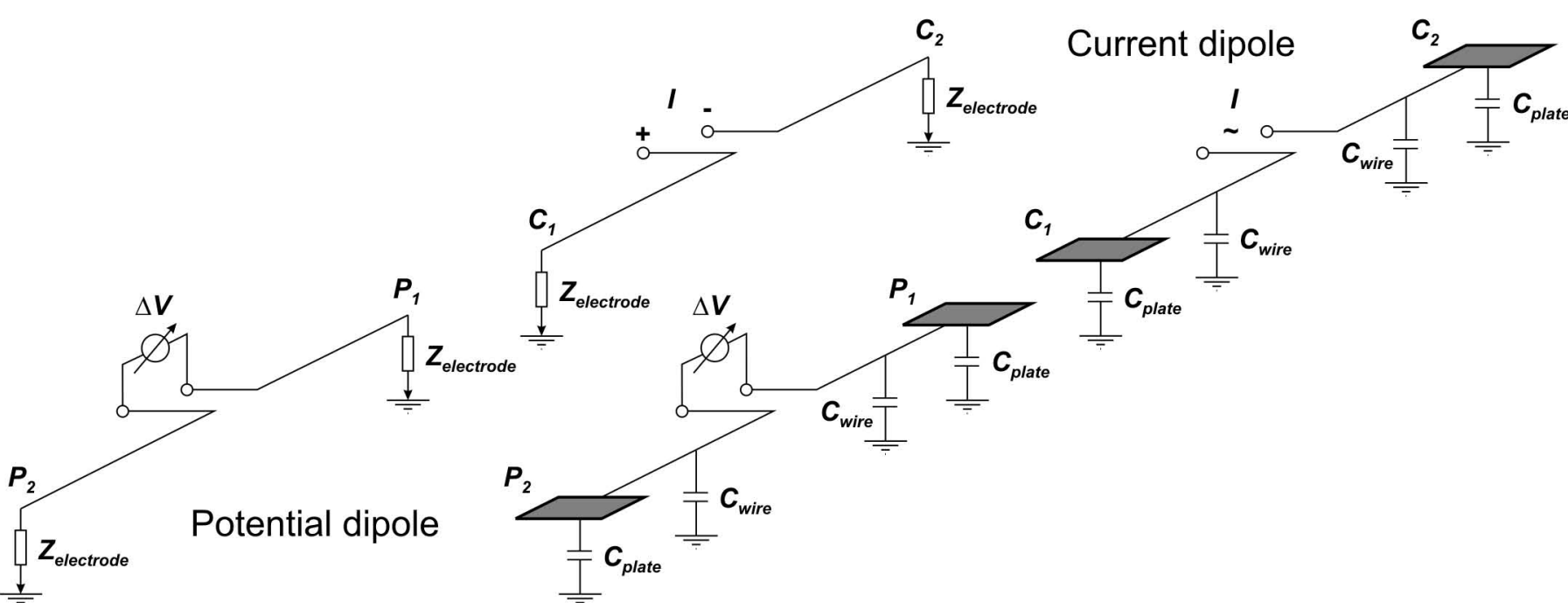


Inline array

\section{Equatorial array}

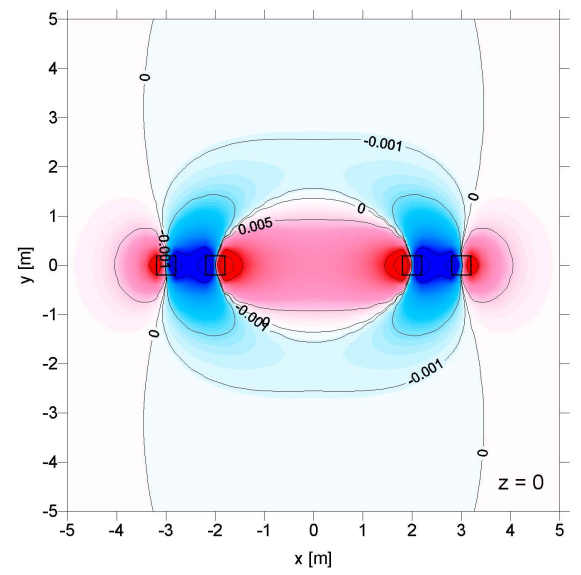

(b) $\frac{E}{N}_{-3}^{-2}$
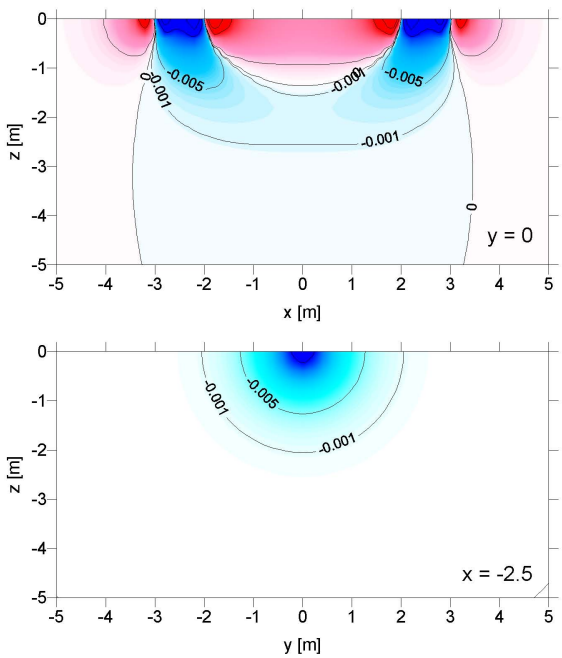
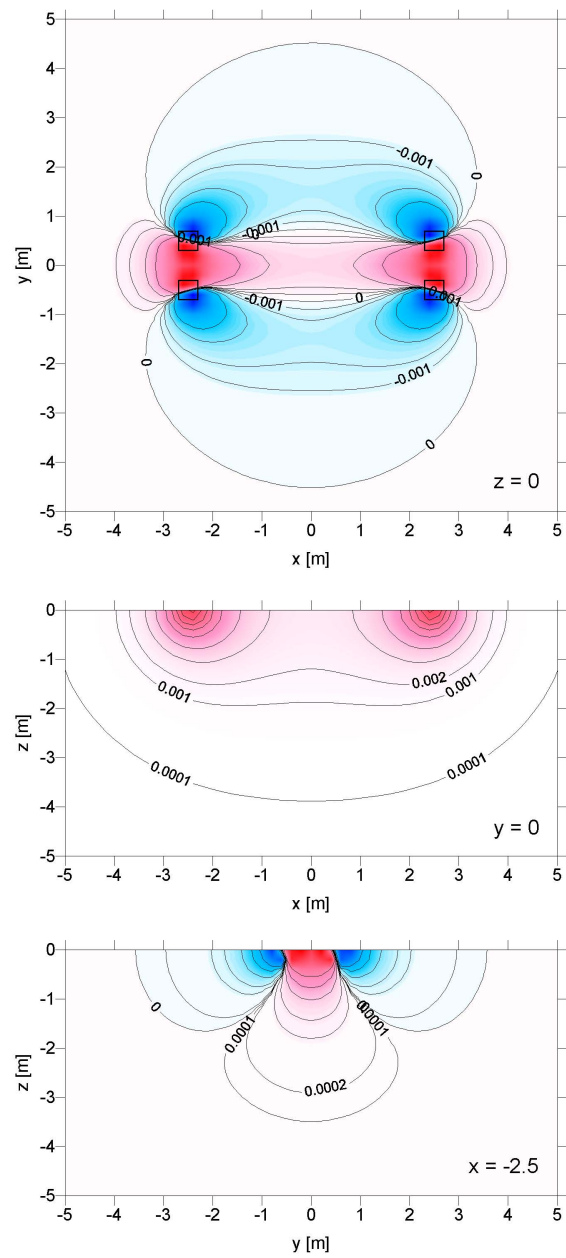
Receiver unit and data acquisition 
(a) In-phase/quadrature

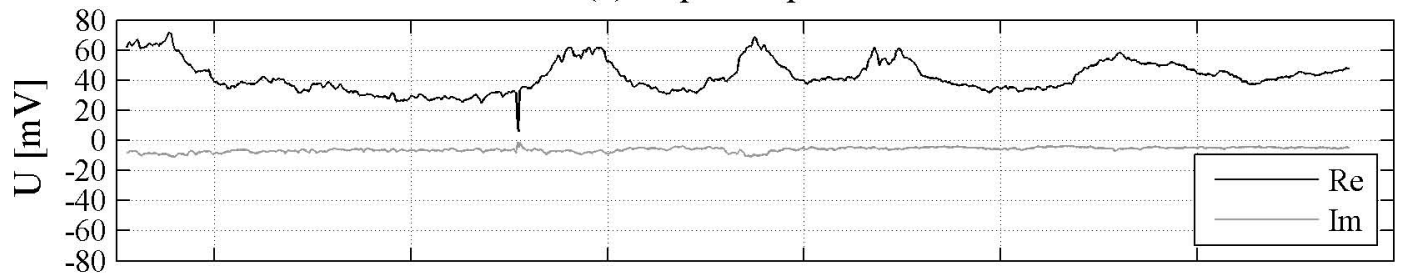

(b) Magnitude

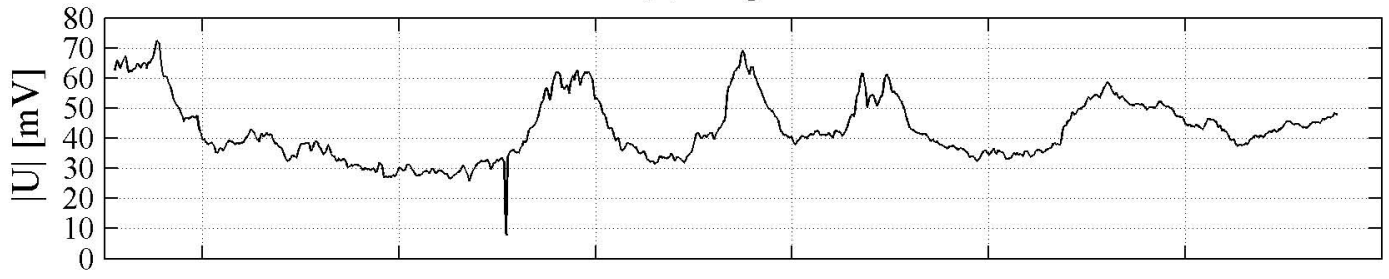

(c) Phase

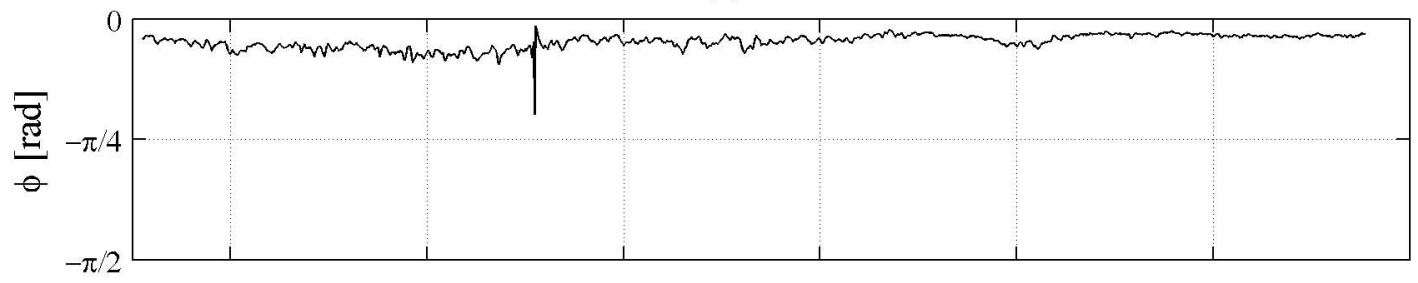

(d) Current

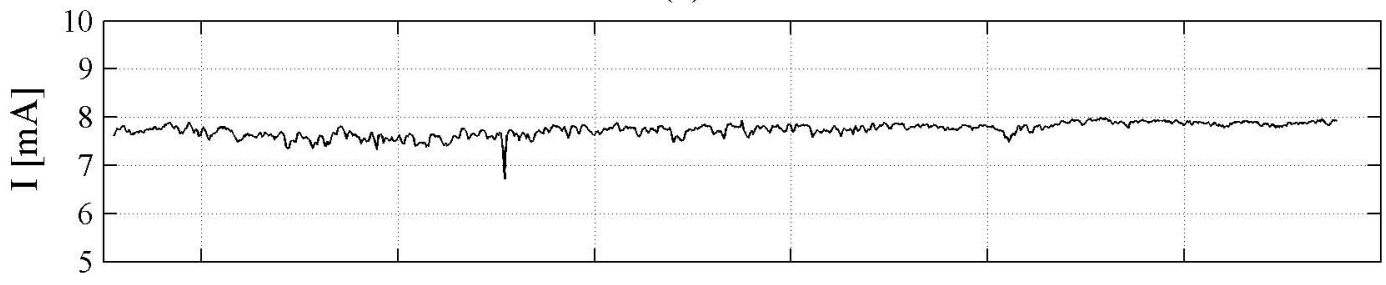

(e) Apparent resistivity

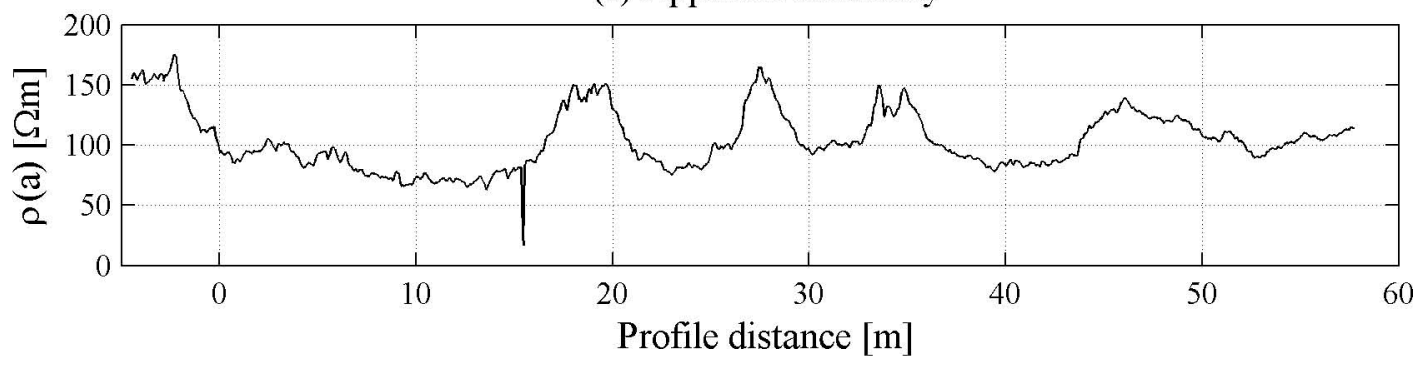




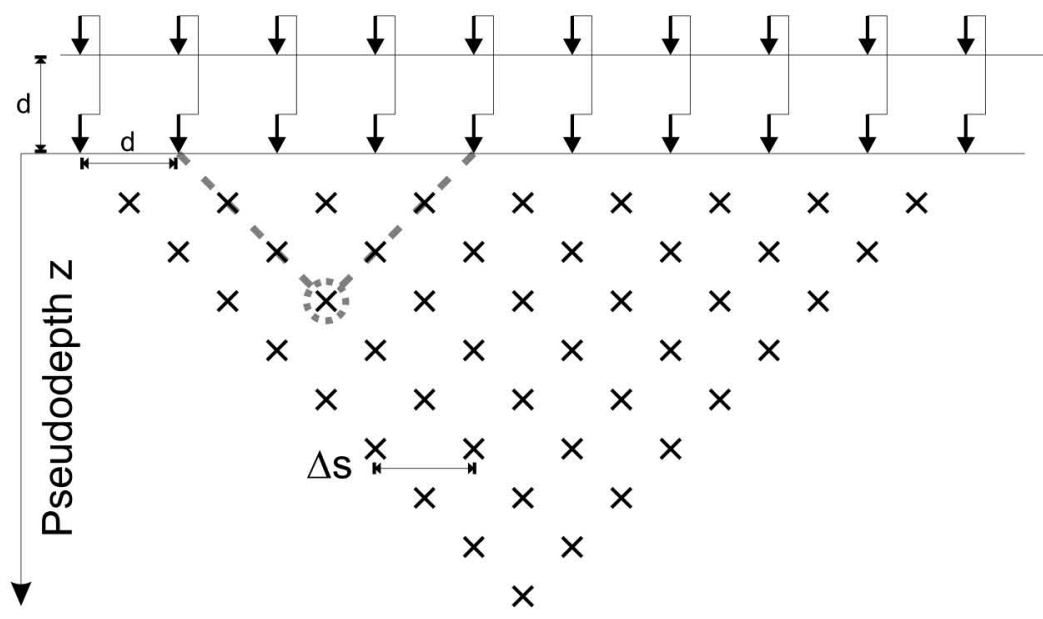

(b)

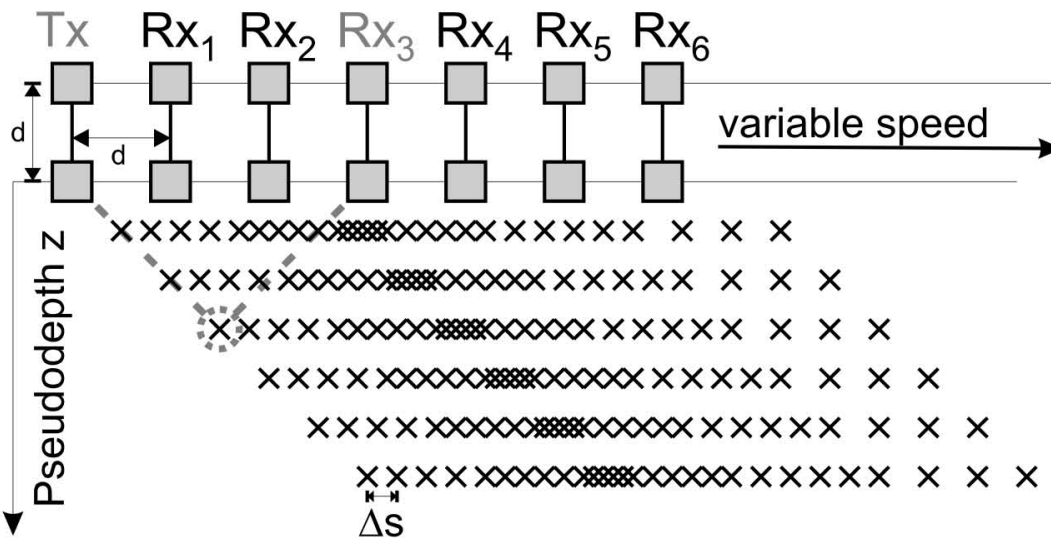

(c)

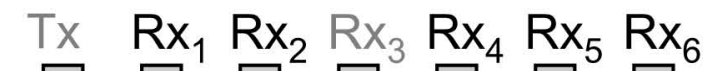

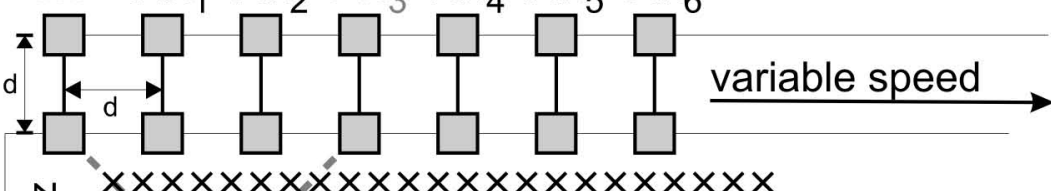

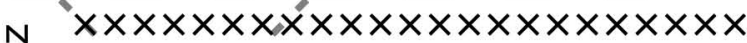

ᄃ $\mathrm{x} \times \mathrm{x} \times \mathrm{x} \times \mathrm{x} \times \mathrm{x} \times \mathrm{x} \times \mathrm{x} \times \mathrm{x} \times \mathrm{x} \times \mathrm{x} \times \mathrm{x}$

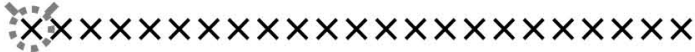

$\times \times \times \times \times \times \times \times \times \times \times \times \times \times \times \times \times \times \times \times \times \times \times$

$\times \times \times \times \times \times \times \times \times \times \times \times \times \times \times \times \times \times \times \times \times \times x$ 

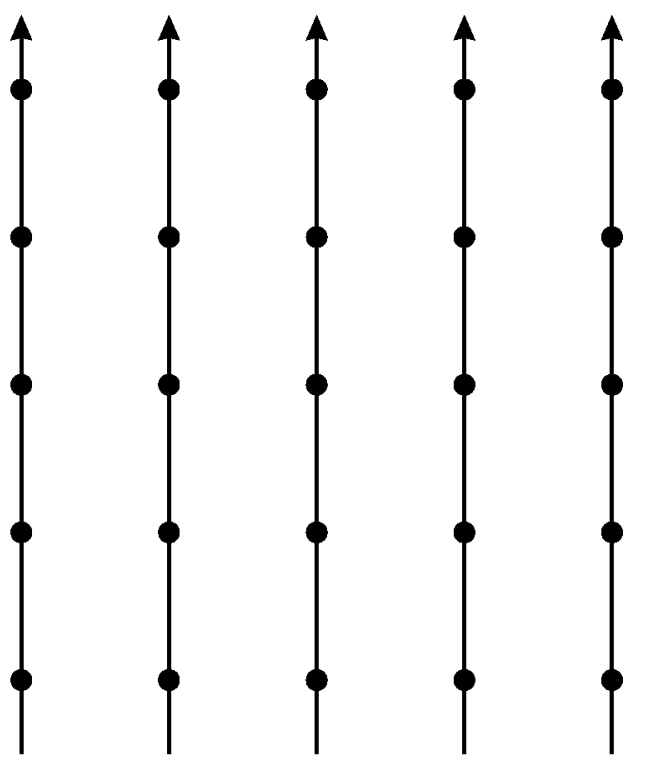

(a)

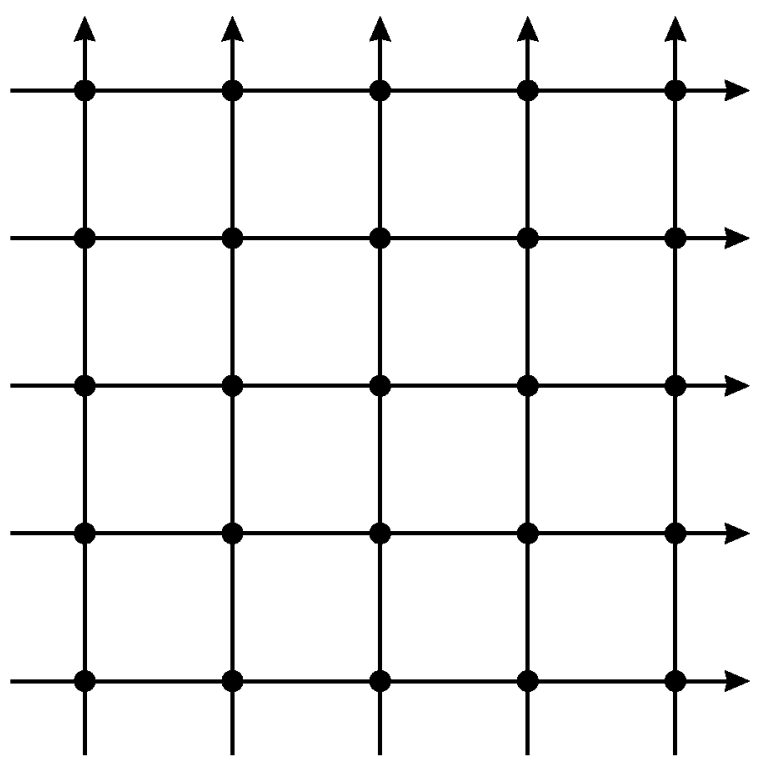

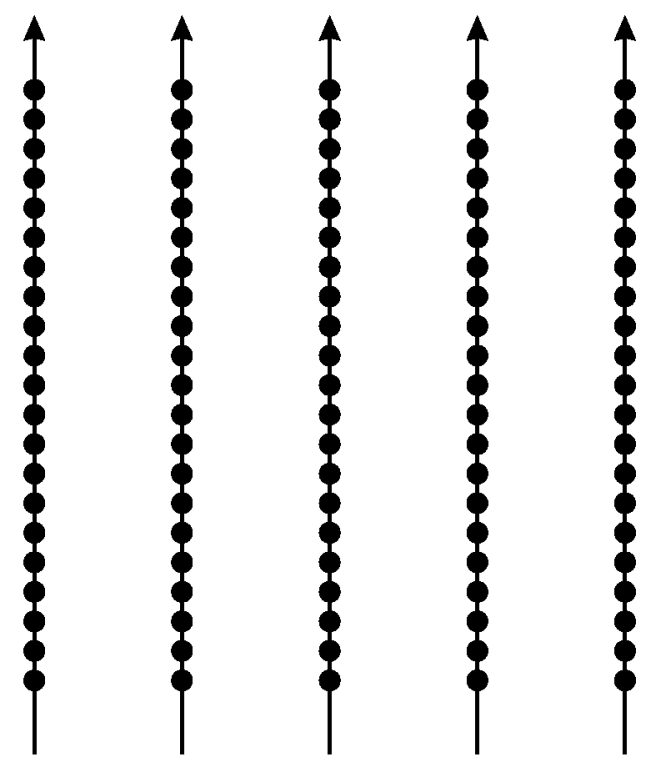

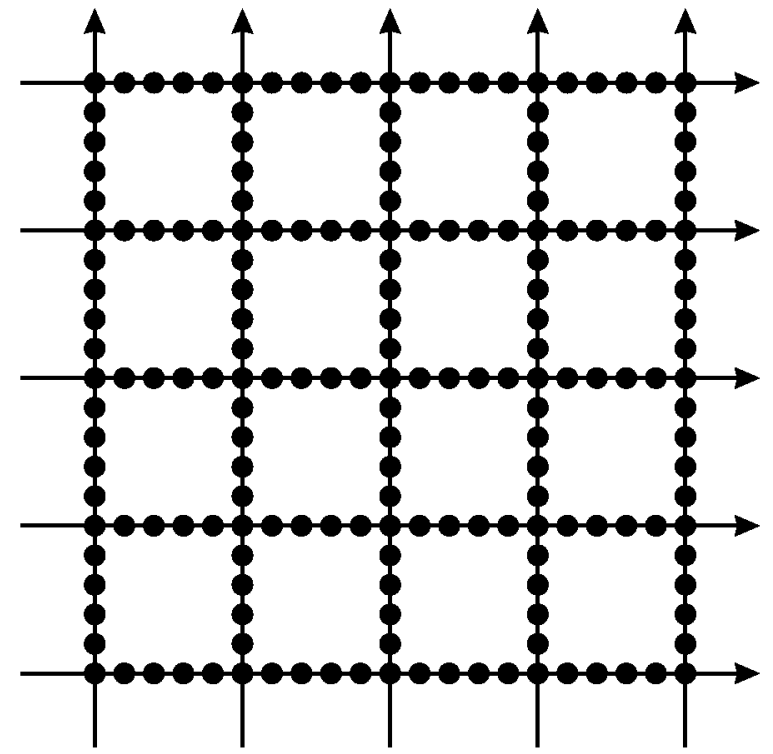

(c)

(d) 
$\mathrm{y}$

Line 1

† • •

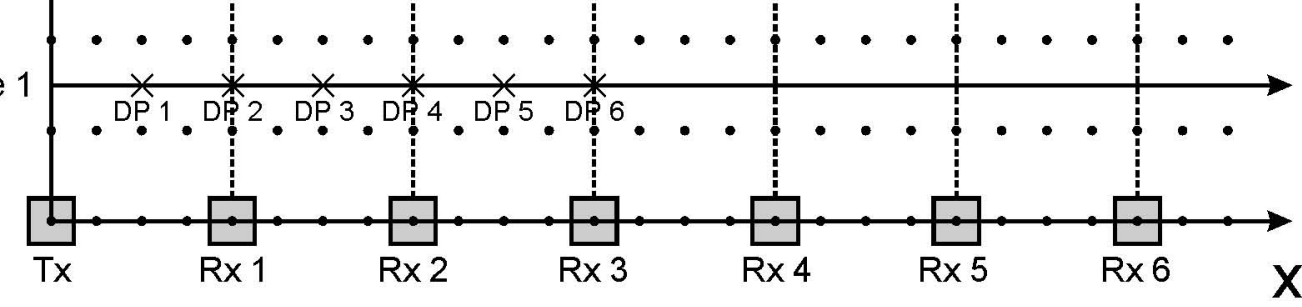

(b)

$\mathrm{y}$

(c)

$\mathrm{y}$

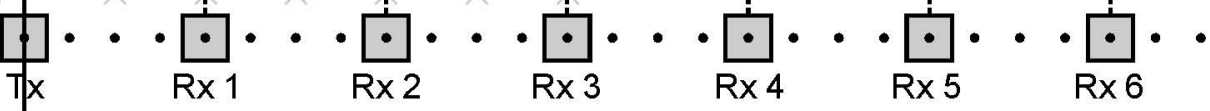

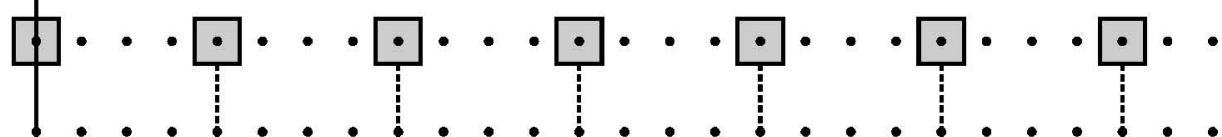

Line 2

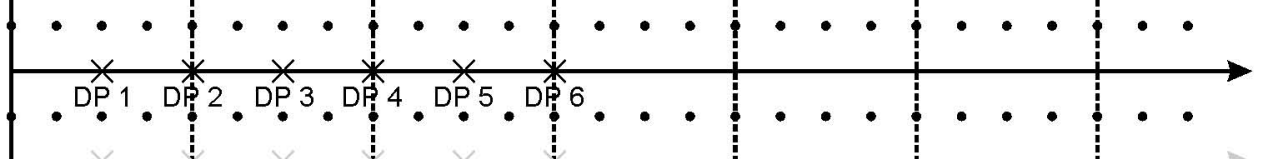

Line 2

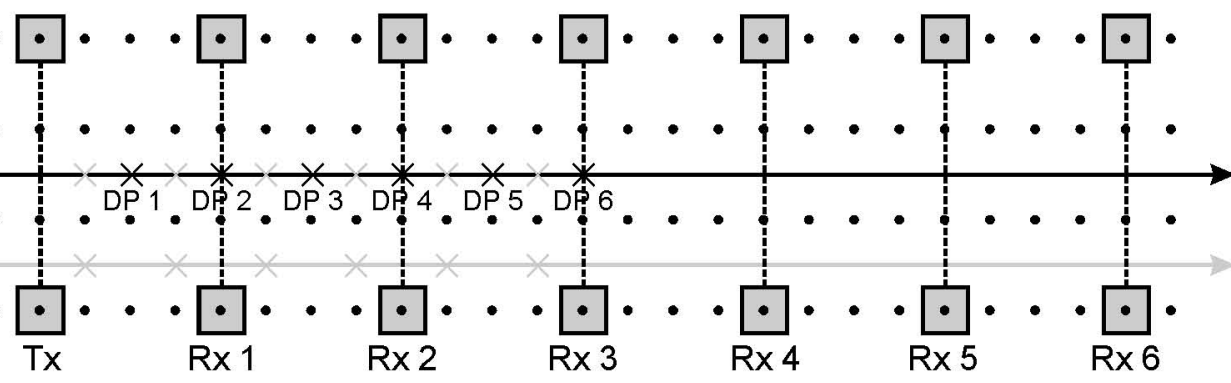



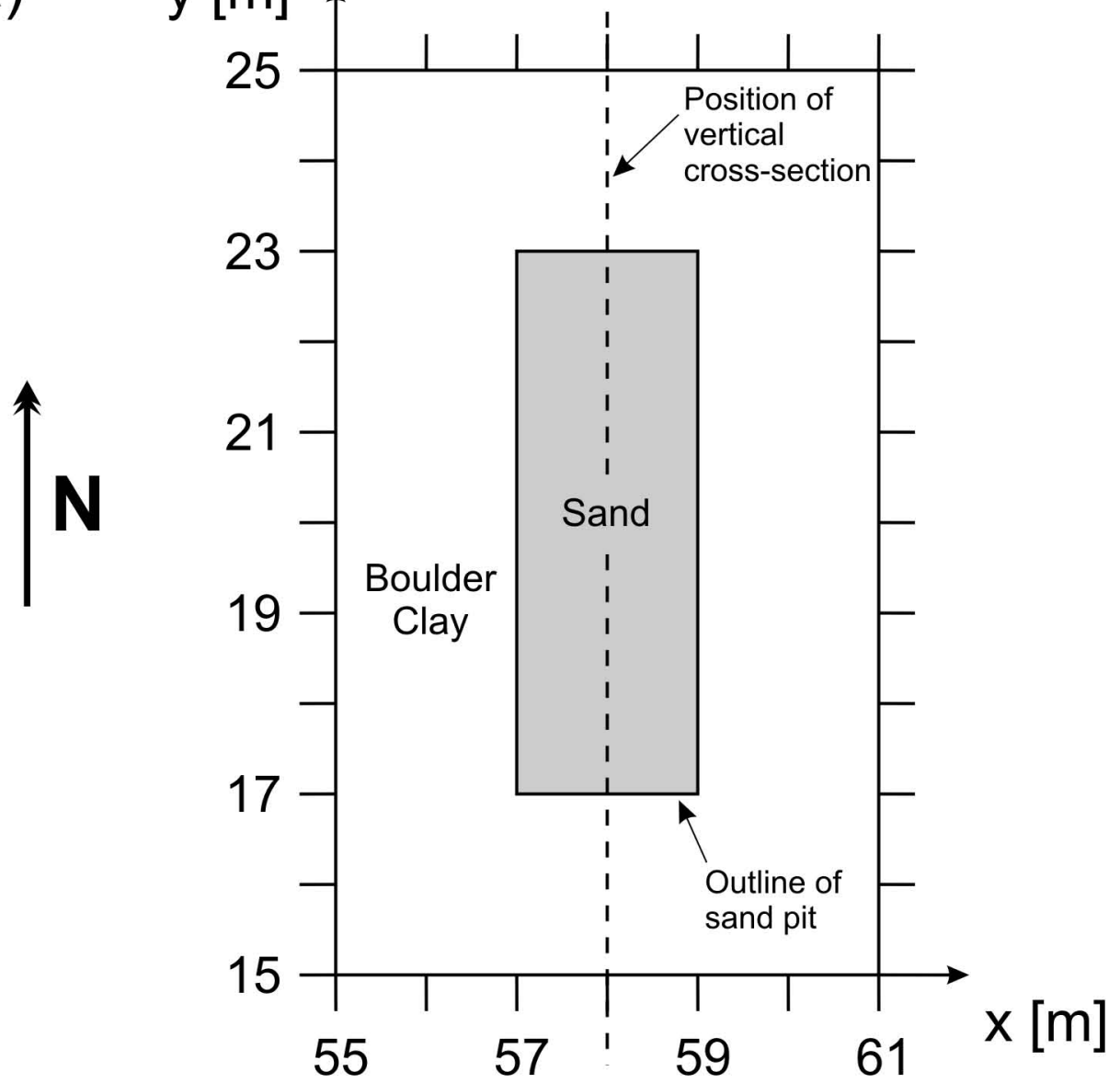

(b)

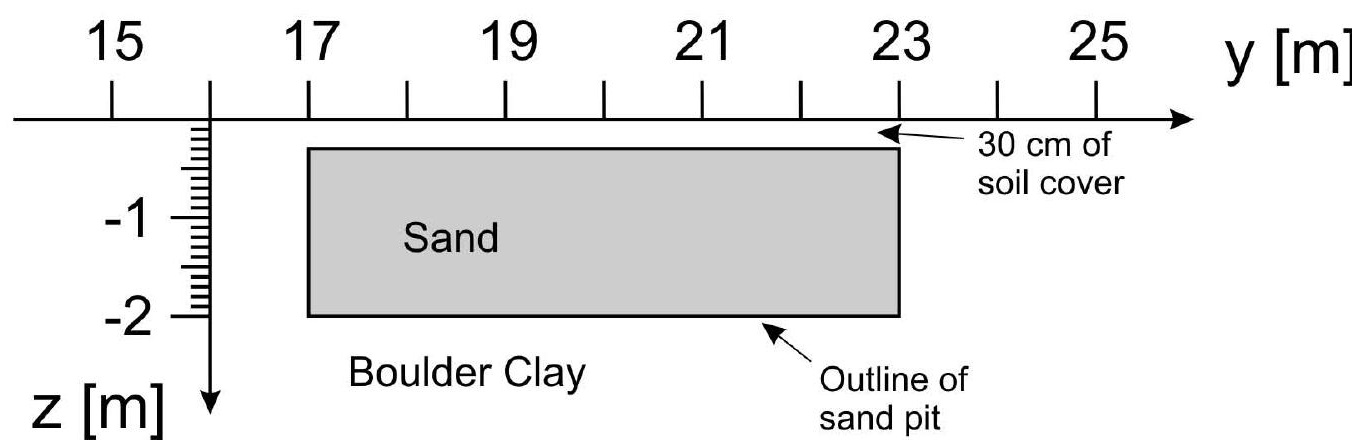




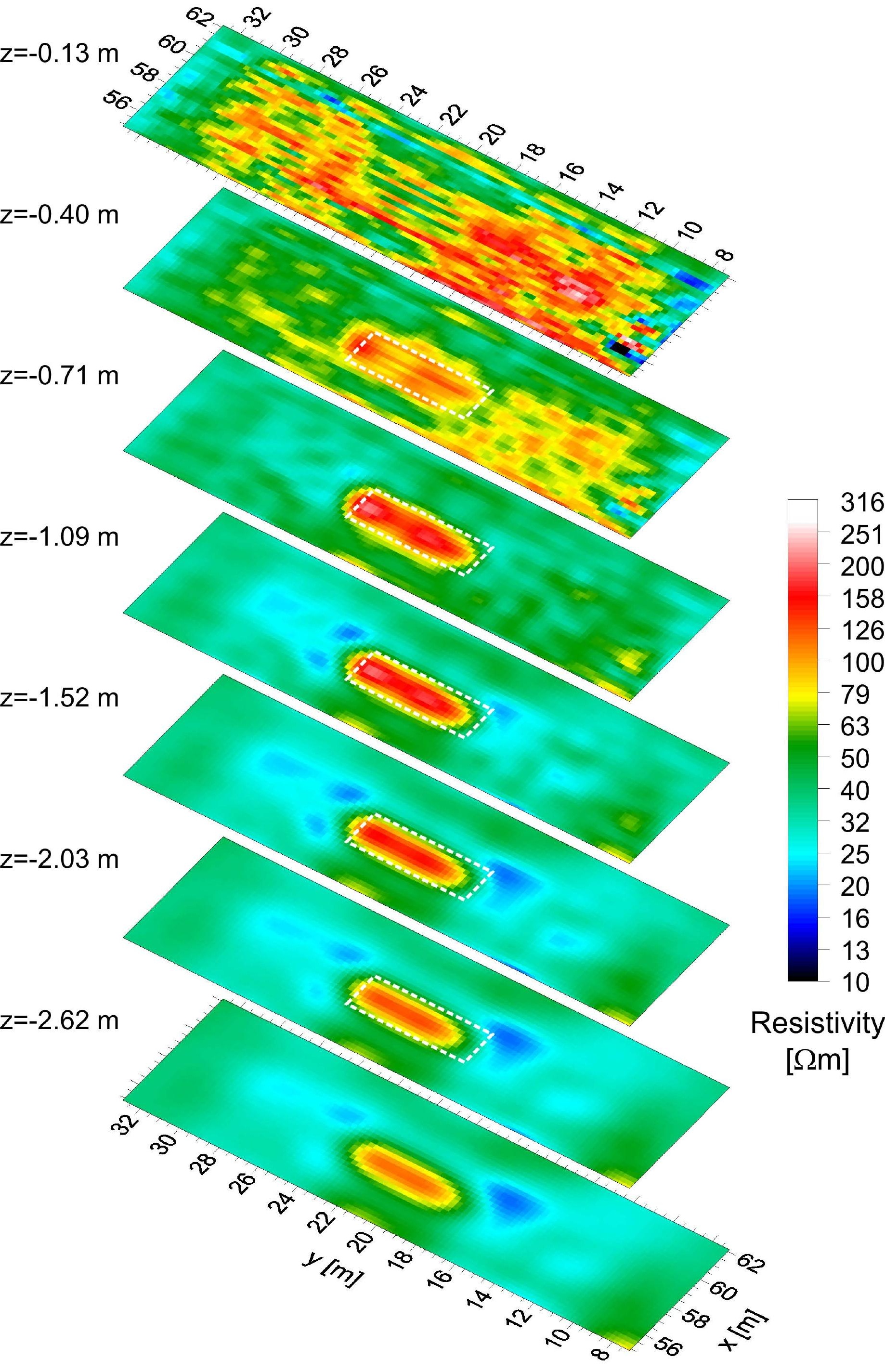

